\title{
Therapeutic role of bone marrow mesenchymal stem cells in diabetic neuronal alternations of rat hippocampus
}

\author{
H.D. Yassa1', S.W. Gergis², L.A. Rashed ${ }^{3}$, D.M. Hassan'1, M.F. Youakim¹ \\ ${ }^{1}$ Department of Anatomy, Faculty of Medicine, Beni-Suef University, Egypt \\ ${ }^{2}$ Department of Anatomy, Kasr El Aini Faculty of Medicine, Cairo University, Egypt \\ ${ }^{3}$ Department of Biochemistry, Kasr El Aini Faculty of Medicine, Cairo University, Egypt
}

[Received: 24 April 2019; Accepted: 2 August 2019]

\begin{abstract}
Background: As the hippocampus is the main brain region for many forms of learning and memory functions and is acutely sensitive to blood glucose changes, diabetes mellitus, which is a serious metabolic disease, is often accompanied by learning and memory deficits. Through scientific literatures, mesenchymal stem cells (MSCS) promote functional recovery in rats with traumatic brain injury, so the present work was conducted to study MSCs as a possible treatment for the diabetic neuronal degeneration and functional impairment of rat hippocampus. Materials and methods: It was carried out using male albino rats: non-diabetic control groups $(4,8,12$ weeks) $(n=15)$, diabetic groups by i.v. injection of streptozotocin for $(4,8,12$ weeks) $(n=15)$ and MSCs treatment to diabetic groups for $(8,12$ weeks) $(n=10)$. Hippocampal learning and memory functions were assessed by the Morris Water Maze test and its results were statistically analysed. The rat hippocampal regions (CA1 and CA3) were subjected to histological, ultrastructural examination and morphometrical analyse of pyramidal neurons. Results: Neurons of the diabetic groups showed disturbed function and architecture; shrunken hyperchromatic nuclei and vacuolated eosinophilic cytoplasm (apoptotic changes) also MSCs treatment improved hippocampal learning and memory functions plus its architectural changes; increasing populations and normal regular distribution.

Conclusions: It can be concluded that diabetic hippocampal neuronal alternations and functional impairment can be ameliorated by MSCs treatment. (Folia Morphol 2020; 79, 2: 211-218)
\end{abstract}

Key words: diabetes, mesenchymal stem cells, hippocampus, neuronal alternations, learning and memory

\section{INTRODUCTION}

Uncontrolled diabetes mellitus (DM) leads to severe complications of central nervous system (CNS) usually associated with neuronal degeneration [31]. As the hippocampus is particularly sensitive to changes in blood glucose level specially CA1 and CA3 regions [21], DM produces hippocampal dysfunction that is involved in learning and memory processing enhancing the risk of Alzheimer's disease [3]. Through scientific literature, DM can affect the hippocampus through reducing its synaptic plasticity [22], changes glutamate neurotransmission [10] and augments enzymatic activity linked to

Address for correspondence: Dr. D.M. Hassan, Department of Anatomy, Faculty of Medicine, Beni-Suef University, Egypt, tel: 002-01003806640, e-mail: doaa_eldary333@yahoo.com 
oxidative stress in the hippocampus [29]. These changes are usually associated with sings of neuronal loss and apoptosis which are due to stimulation of caspase-3 activity, nuclear DNA cleavage and induction of proapoptotic genes in hippocampus especially with uncontrolled DM [19]. As streptozotocin (STZ) can cause pancreatic $\beta$-cell destruction, it is used experimentally to induce type 1 DM [17]. Mesenchymal stem cells (MSCs) have been used experimentally in the treatment of the injured brain $[1,6,11]$ as well as other damaged organs as in kidney [9], heart, etc. In the reviewed literatures there was obvious contradiction concerning the differentiation of MSCs into the functioning nerve cells. Several studies showed that MSCs transplanted into the intact, injured or diseased CNS environments do not differentiate or even a small portion of them produce neural phenotypes [8]. Even though, Chopp and Li [6] demonstrated that, transplanted MSCs promote functional recovery in rats with traumatic brain injury through activation of endogenous angiogenesis, neurogenesis, and synaptogenesis. Moreover, other studies supported that MSCs could differentiate into mature neuron-like cells and exhibit neuronal properties $[1,11]$. Also, Calió et al. [4] discussed MSCs role on the injured brain by decreasing neuronal apoptosis and oxidative stress. The aim of the present work was to evaluate using MSCs as a possible treatment for diabetic neuronal degeneration and learning and memory alternations.

\section{MATERIALS AND METHODS}

\section{MSCs preparation}

Mesenchymal stem cells were obtained from Medical Biochemistry Department, Faculty of Medicine, Cairo University. Bone marrow derived MSCs was isolated and cultivated for 4 weeks according to protocol of Jiang et al. [13]. Next, cells were labelled with 5-bromo-2'-deoxyuridine (BrdU). Fluorescence phase-contrast microscope (Axiocam MR R3, Carl Zeiss, Germany) was used to observe the rat MSCs every 2 or 3 days.

\section{Animals}

The experiment strictly adhered to all ethical guidelines regarding animal research and was approved by the Institutional Animal Care and Use Committee of Cairo University (CU-IACUC). Adult normoglycaemic male Sprague-Dawley albino rats (150-200 g) were used in the current study. Rats were kept in a temperature- and humidity-controlled room, with free access to food and water and were placed 5/cadge.

\section{Experimental design}

The rats were divided into three groups.

Control group ( $n=15)$ which was subdivided into three subgroups ( $n=5 /$ subgroup) [20]: (Control, 4 weeks: sacrificed after 4 weeks, Control, 8 weeks: sacrificed after 8 weeks, and Control, 12 weeks: sacrificed after 12 weeks).

DM groups $(n=15)$ received single i.v. injection of STZ (STZ, Sigma-Aldrich, St. Louis, MO, USA) dissolved in sodium citrate $0.1 \mathrm{~mL}$ buffer, PH 4.5 at a dose of $60 \mathrm{mg} / \mathrm{kg}$ [2]. This group was subdivided into three subgroups ( $n=5 /$ subgroup) [20]: (4-week DM: sacrificed after 4 weeks, 8-week DM: sacrificed after 8 weeks, and 12-week DM: sacrificed after 12 weeks). Fasting blood glucose levels were measured in DM groups (using spectrophotometer-Beckman; USA) $72 \mathrm{~h}$ after STZ injection to confirm induction of DM (> $200 \mathrm{mg} / \mathrm{dL}$ ). This day was considered the first day of the experiment.

DM + MSCs group $(n=10)$ received single i.v. dose of STZ and 4 weeks later a single i.v. dose of fluorescent MSCs $1 \mathrm{~mL}$ of about 2,000,000 stem cells in the rat tail vein [15]. This group was subdivided into two subgroups ( $n=5 /$ subgroup) [20]: (8-week DM + MSCs: sacrificed after 8 weeks from the start of experiment; 4 weeks after MSCs administration and 12-weeks DM + MSCs: sacrificed after 12 weeks from the start of experiment; 8 weeks after MSCs administration). Fasting blood glucose levels were measured in DM and MSCs groups before sacrification.

\section{Morris Water Maze (MWM) test}

Learning and memory impairment induced by DM and improvement after MSCs treatment were evaluated through performing MWM (4, 8 and 12 weeks from the start of the experiment). It was performed according to previous studies by Vorhees and Williams [32]. Four-day training (twice/day) learning and memory was assessed through measuring escape latency to rich hidden platform (recorded with video camera). The maze was built of white circle tank (diameter: $127 \mathrm{~cm}$ and height: $51 \mathrm{~cm}$ ) filled with water depth $45 \mathrm{~cm}$ (made opaque with liquid milk) and a white platform $\left(12 \mathrm{~cm}^{2}\right)$ submerged $2 \mathrm{~cm}$ below the water surface in the northwest quadrant of the maze. Navigational landmarks in the form of external cues were placed to the room wall. Signs of retrieving such memory (by removing platform and animals were allowed to swim freely for $60 \mathrm{~s}$ ) were assessed through a provisional trial by counting both the number of platform-site crossovers and percent- 
age of time spent in the target quadrant compared with the other quadrants. Statistical analysis was done for the means of escape latency times/secs of the trails and for the provisional trial.

\section{Sacrification and histological staining}

By the end of the experimental periods, animals were anaesthetised by i.v. injection of ketamine-dylazine and cardiac perfusion fixation technique by formalin $10 \%$ was done. For brain extraction, animals were decapitated immediately rostral to the first cervical vertebra and brains were removed. The right hemispheres allocated for light microscopic examination stained with haematoxylin and eosin (H\&E). Studying hippocampal both CA1 and CA3 regions, as those regions are the hub of memory and learning functions [18]. The left hippocampi were dissected and allocated for ultrastructural examination using transmission electron microscope JEOL TEM (JEM-1400, JEOL, Japan) at $80 \mathrm{kV}$. To detect labelled MSCs in the hippocampus, 2 sections of both MSCs subgroups were immunostained by anti-Brdu and goat anti-mouse Ig and examined by fluorescent microscopy (Fig. 1).

\section{Morphometric analysis}

It was conducted by using hippocampal histological sections stained by H\&E, implemented for the numbers of the pyramidal neurons in CA1 and CA3 hippocampal regions in all subgroups (5 sections par each) using total magnification of $\times 400$. These statistical assessments were performed using analysis of variance (ANOVA) and statistical package for social science (SPSS). P value $>0.05$ was considered not significant (NS) and $p$ value $<0.05$ was considered significant (S).

\section{RESULTS}

\section{Statistical analysis}

Regarding to the escape latency parameter in MWM test, all control groups showed identical numerical data so they were considered as one control group. All DM subgroups tended to require significant more escape latency periods to find platform than control group and there was more significant difference for 12-week DM subgroup than 8-week DM subgroup ( $<<0.05)$ (Fig. 2A). On the other hand, MSCs treatment improved learning and memory deficit, as both MSCs subgroups exhibited a significant decrease in the escape latency period $(p<0.05)$ as

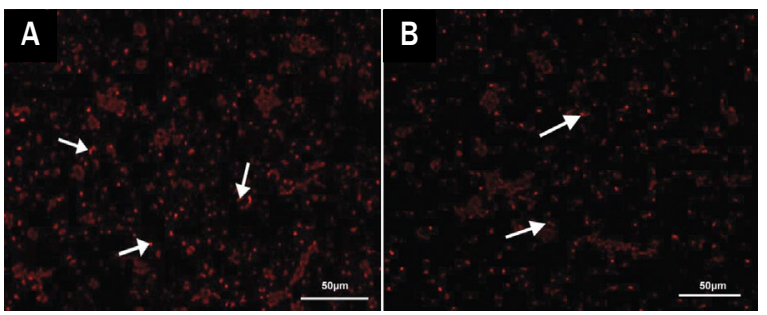

Figure 1. Hippocampal fluorescent-labelled mesenchymal stem cells (MSCs), immunostained with 5-bromo-2'-deoxyuridine (BrdU) antibody in 8-week DM+MSCs group (A) and 12-week DM + MSCs group (B); bar $=50 \mu \mathrm{m}$; DM - diabetes mellitus.

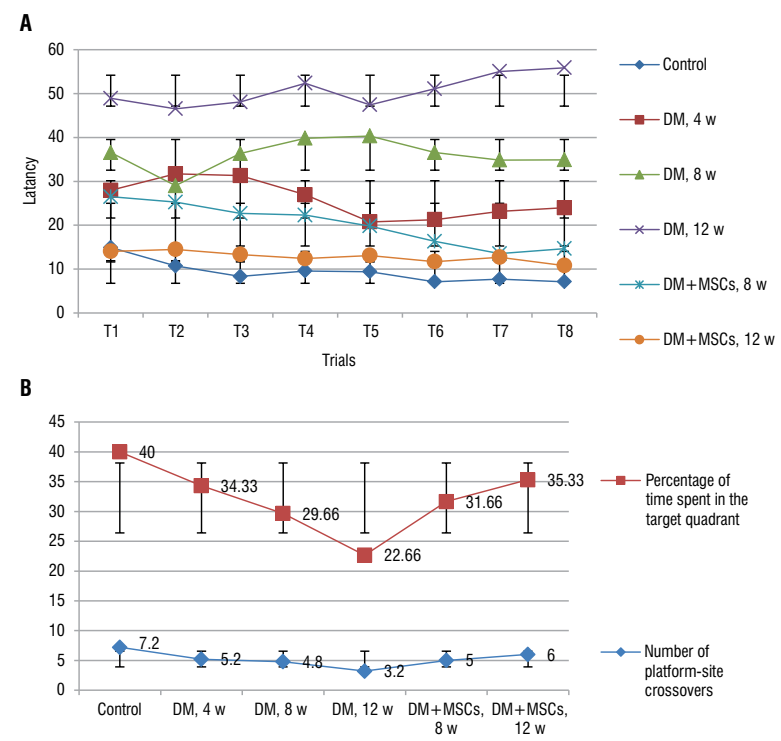

Figure 2. A. The means of escape latency periods during all Morris Water Maze training trials in all subgroups; $\mathbf{B}$. The provisional trial results (the mean numbers of the platform-sites crossovers and the percentage of time spent in the target quadrant) in all subgroups; DM — diabetes mellitus; MSCs - mesenchymal stem cells; w - weeks.

compared to DM subgroups (Fig. 2A) but still significantly higher numbers when compared to control groups ( $p<0.05$ ) (Fig. 2A). Evaluation of provisional trial test showed the mean values of the DM subgroups were significantly lower compared to control groups ( $p<0.05$ ). However, the outcomes of DM + MSCs subgroups were significantly improved when compared to DM subgroups, but still lower than that of control groups $(p<0.05)$ (Fig. 2B).

Concerning fasting blood glucose levels in all groups, the mean values were significantly increased in DM groups according to the extent of diabetic durations $(p<0.05)$ but with MSCs treatment the values were significantly decreased according to the extent of treatment indicating improvement $(p<0.05)$ (Table 1). 
Table 1. The mean values of fasting blood glucose level $(\mathrm{mg} / \mathrm{dL})$ in all groups

\begin{tabular}{cccccc}
\hline $\begin{array}{c}\text { Control } \\
\text { groups }\end{array}$ & DM, 4 w & DM, 8 w & DM, 12 w & $\begin{array}{c}\text { DM + } \\
\text { MSCs, } \\
\mathbf{8 ~ w ~}\end{array}$ & $\begin{array}{c}\text { DM + } \\
\text { MSCs, } \\
\mathbf{1 2 ~ w ~}\end{array}$ \\
\hline $82.7 \pm$ & $224 \pm$ & $248.5 \pm$ & $273 \pm$ & $134.5 \pm$ & $117 \pm$ \\
5.65 & 6.91 & 35.08 & 13.93 & 22.59 & 7.91 \\
\hline
\end{tabular}

DM — diabetes mellitus; MSCs — mesenchymal stem cells; w — weeks

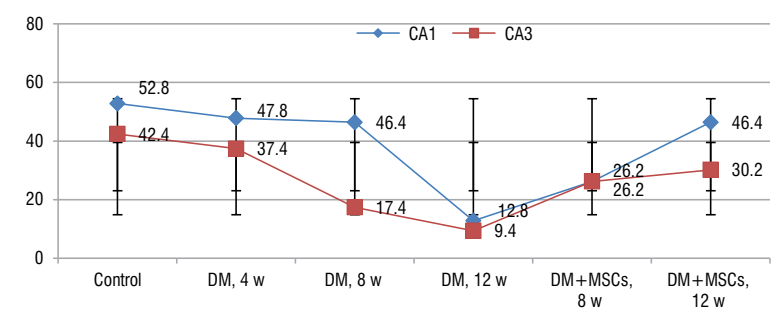

Figure 3. The mean numbers of the pyramidal neurons in regions (CA1 and CA3) in all subgroups; $w$ - weeks.

Regarding to the morphometrical statistical analyses of the pyramidal neurons, mean values were used to assess diabetic neuronal degeneration and regeneration by MSCS of CA1 and CA3 regions.

The numbers of both regions of DM subgroups were decreased when compared to control group. Pyramidal cell reduction of CA3 was significant in 8-week and 12-week DM subgroups while the reduction of number of cells of CA1 was only significant in 12 -week DM subgroup $(p<0.05)$. Moreover, among DM subgroups, their mean numbers were decreased according to the extent of DM duration; this reduction was significant in rats of 12 -week DM subgroup $(p<0.05)$. There was prominent neuronal number improvement in DM + MSCs subgroups. However, the values were still significantly lower in 8-week DM + MSCs subgroup when compared to control group $(p<0.05)$. On the other hand, marked improvement in their numbers was demonstrated in rats of 12-week DM + MSCs subgroup which was non-significant in CA1 region and only significant in CA3 region when compared to the control group ( $p>0.05$ ) (Fig. 3).

\section{Haematoxylin and eosin stained sections}

All control groups showed identical histological architecture, so they were considered as one control group.

Examination of CA1 region. The control group revealed arranged pyramidal neurons of uniform size and shape with narrow neuropil in-between, each en-

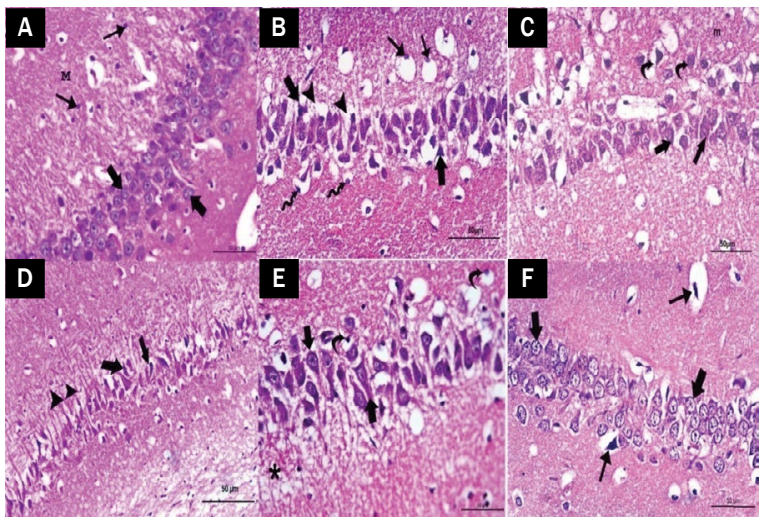

Figure 4. CA1 region; A. Control group: arranged pyramidal $(\mathrm{P})$ with vesicular nucleus (thick arrows) and glial cells (thin arrows) in molecular layer (M); B. Four-week DM: karyorrhectic (thick arrows), karyolytic (zigzag arrow) and shrinkage nuclei (thin arrows), vacuolations (arrowheads); C. Eight-week DM: clogged marginated chromatin (arrows) and hyperchromatic neurons (curved arrows) in molecular layer (M); D. Twelve-week DM: severe degeneration as, many shrunken nuclei (arrows) and clumping neuronal processes (arrowheads); E. Eight-week DM + MSCs: neuronal improvement (thick arrows), few shrunken nuclei (curved arrows) and clumping processes (asterisk); $\mathbf{F}$. Twelve-week DM + MSCs: progressive neuronal improvement (thick arrows), few shrunken neurons (thin arrows); bar $=50 \mu \mathrm{m}$; $\mathrm{DM}$ — diabetes mellitus; MSCs — mesenchymal stem cells.

closing single, rounded, large and vesicular nucleus with prominent nucleolus. Many glial cells within the molecular layer were seen (Fig. 4A). On examination of 4-week DM subgroup, most of the pyramidal neurons were comparable to the control, while other few neurons were shrunken with karyorrhectic and karyolytic nuclei. Vacuolations were also seen (Fig. 4B). On examination of the 8-week DM subgroup showed more neuronal damage as many neurons had clogged marginated chromatin or hyperchromatic neurons (Fig. 4C). Moreover, on examination of 12-week DM subgroup exhibited severe damage as apparent losses of the neuronal regularity: many shrunken hyperchromatic nuclei and severe clumping of the neuronal processes (Fig. 4D). On the other hand on examination of 8-week $\mathrm{DM}+\mathrm{MSC}$ subgroup, there were variable degrees of improvement as neurons seemed normal comparable to the control: relatively few shrunken nuclei with vacuolated cytoplasm and small areas with clumping neuronal processes (Fig. 4E). Moreover, examination of 12-week DM + MSCs subgroup demonstrated more improvement as increased population of pyramidal neurons with apparent compact and regular distribution, few shrunken neurons with hyperchromatic nuclei (Fig. 4F).

Examination of CA3 region. The control group revealed arranged layers of pyramidal neurons with vesicular nuclei and well-formed basophilic cytoplas- 


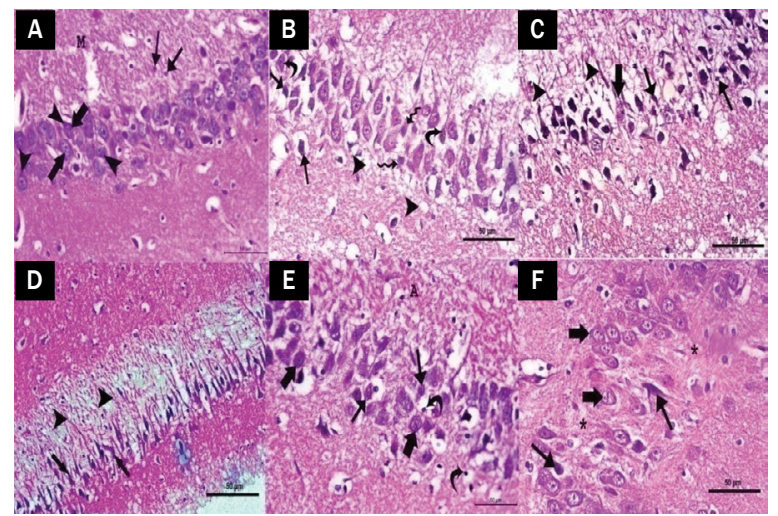

Figure 5. CA3 region; A. Control group: arranged pyramidal neurons (thick arrows), well-formed Nissl granules (arrowheads) and glial cells (thin arrows) in the molecular layer (M); B. Four-week DM: clogged marginated nuclear chromatin (curved arrows), karyorrhectic nuclei (zigzag arrows), vacuolated cytoplasm (thin arrows) and neuropil vacuolations (arrowheads); C. Eight-week DM: pyknotic nuclei (thin arrows), neuron with homogenous nucleus (thick arrow) and clumped processes (arrowheads); D. Twelveweek DM: more degeneration as eosinophilic cytoplasm (thick arrows) and clumping processes (arrowheads); E. Eight-week DM + MSCs: minimal regeneration as neuronal vesicular nuclei (thick arrows), karyolytic (thin arrows) or pyknotic nuclei (curved arrows) and amyloid depositions (A); F. Twelve-week DM + MSCs: more regeneration as pyramidal neurons with vesicular nuclei (thick arrows), areas devoid of neurons (asterisks) and few shrunken neurons (thin arrows); bar $=50 \mu \mathrm{m} ; \mathrm{DM}-$ diabetes mellitus; MSCs - mesenchymal stem cells.

mic Nissl granules surrounded by narrow neuropil. The molecular layer showed glial cells (Fig. 5A). However in 4-week DM subgroup, the pyramidal neurons represented normal nuclear appearance, while few nuclei with clogged marginated chromatin, relatively few shrunken neurons with vacuolated cytoplasm or with peripheral karyorrhectic nuclei and narrow neuropil with few vacuolations (Fig. 5B). Moreover, on examination of 8-week DM subgroup there were pleopathological changes, such as pyramidal neurons haphazardly arranged with many pyknotic nuclei, neurons with peripheral homogenous nucleus and severe vacuolation with clumping processes (Fig. 5C). Also, the 12-week DM subgroup showed more degeneration reflected by neuronal disorganisation with eosinophilic vacuolated cytoplasm and severe clumping processes (Fig. 5D). While on examination of 8-week DM + MSCs subgroup, there was minimal regeneration: pyramidal neurons showed normal appearance and almost normal neuropil comparable to control group. However, others were represented with karyolytic or pyknotic nuclei and vacuolated cytoplasm. Extraneuron amyloid deposition was noticed (Fig. 5E). Additionally, 12-week DM + MSCs subgroup

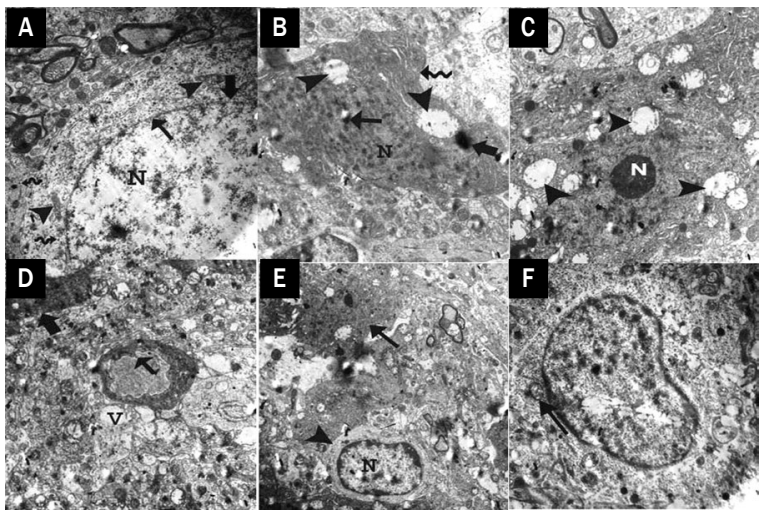

Figure 6. Pyramidal neurons; A. Control group: normal neuron with intact mitochondria (arrowheads), lysosomes (zigzag arrow), euchromatic nucleus (N), smooth envelope (thin arrow) and wellformed nuclear pores (thick arrow); bar $=2 \mu \mathrm{m}$; B. Four-week DM subgroup: irregular neuronal out line (zigzag arrow), ballooning mitochondria (arrowheads), electron-dense body (thick arrow), the nucleus (N) with chromatin clumps (thin arrow); bar $=2 \mu \mathrm{m}$; C. Eight-week DM subgroup: more degeneration as nuclear condensation (N), many ballooning mitochondria (arrowheads); bar $=2 \mu \mathrm{m}$; D. Twelve-week DM subgroup: severe degeneration as condensation with membrane indentation (thin arrow), highly condensed dense chromatin neuron (thick arrow) and vacuolations (V); bar $=2 \mu \mathrm{m}$; E. Eight-week DM + MSCs subgroup: some regeneration as normal neuronal form (arrowhead) with euchromatic nucleus (N), few abnormal dense neurons (arrow); bar $=2 \mu \mathrm{m}$; $\mathbf{F}$. Twelve-week DM + MSCs subgroup: more regeneration as normal neuronal ultra-structures with intact mitochondria (arrow); bar $=2 \mu \mathrm{m}$; DM — diabetes mellitus; MSCs - mesenchymal stem cells.

had regeneration signs as most of the pyramidal neurons were comparable to control with vesicular nuclei. Only few shrunken neurons represented with hyperchromatic nucleus and areas devoid of neurons were seen (Fig. 5F).

\section{Ultrastructural study of the pyramidal neurons}

The control groups revealed the normal neuronal ultrastructure; intact cell membrane, intact cytoplasmic mitochondria with normal cristae. Euchromatic nucleus with smooth nuclear envelope consisting of bilaminar layers and well-formed nuclear pores were seen (Fig. 6A). On the other hand, the pyramidal neurons of 4-week DM subgroup showed dense neuron form with irregular out line, ballooning mitochondria with disrupted cristae and cytoplasmic deposition of electron-dense bodies. The nucleus showed rounded and distinctly chromatin clumps (Fig. 6B). Pyramidal neurons of 8-week DM subgroup had more degeneration, such as nuclear condensation, ballooned mitochondria and more cytoplasmic lipofuscin particles (Fig. 6C). Additionally, pyramidal neurons of 12-week DM subgroup had severe degeneration signs 
and apoptotic changes, such as nuclear membrane indentation, cytoplasmic condensation, few neurons with highly condensed dense chromatin and vacuolations within the neuropil (Fig. 6D). While on examination of pyramidal neurons of 8-week DM + MSCs subgroup, there were some evidence of regeneration, such as normal neuronal form with euchromatic, central, and rounded nuclei. But few cells had abnormal dense form with shrunken and electron dense nuclei (Fig. 6E). Moreover, in pyramidal neurons of 12-week DM + MSCs subgroup, there was more evidence of regeneration, such as normal neuronal ultrastructure with intact mitochondria (Fig. 6F).

\section{DISCUSSION}

Diabetes mellitus is a common serious metabolic disease and usually associated with cerebral alternations [31]. Several studies indicated that DM negatively affects hippocampal cellular morphology, proliferation and survival, indicating function impairment of this brain region [35]. MSCs treatment had been shown as a promising modality in the treatment of the injured brain [11], beside that there were contradictions concerning the differentiation of MSCs into the functioning nerve cells. In the present study, the MWM results of DM subgroups indicating learning and memory impairment which become worst with prolonged durations. Similar results were obtained by Malone et al. [23] who further explained that DM decreased hippocampal neurotransmitters' release: presynaptic synaptophysin and postsynaptic density protein, which is the cause of memory and learning deficiency. However in MSCs treated subgroups, there was prominent functional improvement which got better with prolonged treatment duration, indicating progressive improvement of neuronal function. These results were in agreement with the findings of Chen et al. [5] who detected that MSCs increase production of neurotrophic factors and enhance neuronal plasticity of the injured brain.

Alongside with these functional impairment and improvements, the histological and ultrastructural examination showed hippocampal degenerative changes accompanying DM were variable regarding the affected region and also related to the duration of DM exposure. Minor apoptotic alternations were detected in 4-week DM subgroup in both CA1 and CA3 regions, in agreement with findings Zhao et al. [36] who study CA1 and dentate gyrus. However, more changes were mainly seen in CA3 region of 8-week DM subgroup concurring to findings of Jafari et al. [12] and Kamal et al. [14]. Furthermore, when DM was prolonged for 12 weeks, progressive changes were demonstrated mainly in CA3 region. These results were in harmony with findings of long-term study of Yang et al. [33].

These apoptotic degenerative changes were confirmed by more accurate statistical morphometric data as all DM subgroups showed reduction of number of pyramidal neurons which was more significant at 12 weeks than 4 weeks and 8 weeks indicating the progressive degenerative changes.

The findings of earlier diabetic neuronal apoptotic changes detected in the current work were explained by Zhao et al. [36] and Orlovsky et al. [27] as excessive production of nitric oxide in the hippocampal areas which has neurotoxic effect inducing neuronal damage and apoptosis. Also, the findings of progressive diabetic neuronal apoptotic changes were further explained by Yang et al. [33] who established that long-term DM induces oxidative stress and lipid peroxidation with impairment of membrane functions. This occurs through decreasing membrane fluidity and changing the activity of membrane-bound enzymes and receptors. Additionally, Li et al. [19] clarified neuronal cells' apoptosis, as diabetic hyperglycaemia leads to opening of mitochondrial permeability transition pores which directs up expression of caspase-3 and caspase- 8 that play a central role in the execution-phase of cell apoptosis.

Seeking for potential improving role of MSCs, fluorescent-labelled MSCs were recognised in the hippocampus after their systemic injection according to each DM + MSCs subgroups. In the current work, the histological and ultrastructural examination of MSCs treated subgroups showed variable amelioration of the pathological changes and increase in neuronal numbers regarding the extent of MSCs treatment and degree of damage produced by DM on the hippocampus. MSCs treatment for 4 weeks after 4 weeks of diabetic neuronal damage led to improvement of apoptotic changes which was more evident in CA1 region than in CA3 region. Moreover, this improvement was more apparent with prolonged duration of MSCs treatment to 8 weeks after 4 weeks of diabetic neuronal damage. There was obvious regression of neuronal apoptotic changes of CA1 and CA3 with relatively normal pyramidal neuronal structure.

Those histological changes were further documented by the statistical analysis of the morphometric counting of pyramidal neurons of both regions. 
There was increase in neuronal numbers especially with prolonged MSCs treatment in both regions, but this increase was still lower than in control groups. In the reviewed literature, no studies examined the effect of i.v. MSCs administration on hippocampal neuronal diabetic degeneration. However, the MSCs induced neuronal improvement of CA1 region after short-term treatment observed in the current work corresponds to findings reported by Kumar et al. [16] who studied cold stress as a cause of hippocampal neuronal degeneration. Also, the improvement found after longer duration of MSCs treatment was supported by the results obtained by Ye et al. [34] who studied the neuronal degeneration in Parkinson disease, as there was neuronal recovery after 10 weeks of MSCs transplantation. While, the moderate improvement observed in the current work in CA3 region was in harmony with the findings of Matchynski-Franks et al. [24] who studied MSCs effect after 10 weeks of transplantation in a mouse model of Alzheimer's disease.

Discussing the improving role of MSCs in the current study to ameliorate diabetic neuronal degeneration and functional impairment, many authors explained their effects in the injured brain. Mezey et al. [25] concluded that human MSCs could enter the human and rat brains and generating neurons especially in the hippocampus and cerebral cortex. Moreover, Zhang et al. [35] found that transplanted MSCs could engraft into brain tissue and differentiate in vivo into neurons and glial cells. In contradiction, Sanchez-Ramos et al. [30] described that a small proportion of MSC-derived cells differentiated into neuron-like cells. Those findings were explained by Crigler et al. [7] and Munoz et al. [26] as MSCs implanted into the hippocampus did not proliferate, but they greatly increased proliferation, migration and differentiation of the endogenous neuronal stem cells after 8 weeks of neuronal degeneration. Prockop [28] described that MSCs could promote the neurogenesis of primary neural progenitors and survival of neural cells by expressing neurotrophic factors. Also, Calió et al. [4] reported that MSCs could decrease neuronal oxidative stress and apoptosis changes by enhancing the anti-apoptotic activity and antioxidant protection. These results were very promising for the development of a new therapeutic strategy for patient exhibiting diabetic neuronal degeneration and learning and memory impairment by using MSCs treatment.

\section{CONCLUSIONS}

Administration of MSCs ameliorates diabetic neuronal degeneration and learning and memory impairment mainly through promoting neurogenesis, alleviating oxidative stress and also through its anti-apoptotic effects. So, treatment with bone marrow-derived MSCs may exert a successful curative role in patients suffering from diabetic neuronal complications and improving their quality of life.

\section{Acknowledgements}

We are grateful to lecturer Dr. Shaimaa Senosy, Public Health and Community Medicine Department, Beni-Suef University, Egypt for providing help in the statistical analysis.

\section{REFERENCES}

1. Alexanian AR, Maiman DJ, Kurpad SN, et al. In vitro and in vivo characterization of neurally modified mesenchymal stem cells induced by epigenetic modifiers and neural stem cell environment. Stem Cells Dev. 2008; 17(6): 1123-1130, doi: $10.1089 / \mathrm{scd} .2007 .0212$, indexed in Pubmed: 18484898.

2. Bree A, Puente E, Daphna-lken $D$, et al. Diabetes increases brain damage caused by severe hypoglycemia. Am J Physiol-Endocrinol Metabolism. 2009; 297(1): E194-E201, doi: 10.1152/ajpendo.91041.2008, indexed in Pubmed: 19435850.

3. Biessels GJ, van der Heide LP, Kamal A, et al. Ageing and diabetes: implications for brain function. Eur J Pharmacol. 2002; 441(1-2): 1-14, doi: 10.1016/s00142999(02)01486-3, indexed in Pubmed: 12007915.

4. Calió ML, Marinho DS, Ko GMi, et al. Transplantation of bone marrow mesenchymal stem cells decreases oxidative stress, apoptosis, and hippocampal damage in brain of a spontaneous stroke model. Free Radic Biol Med. 2014; 70: 141-154, doi: 10.1016/j.freeradbiomed.2014.01.024, indexed in Pubmed: 24525001.

5. Chen J, Li Yi, Katakowski M, et al. Intravenous bone marrow stromal cell therapy reduces apoptosis and promotes endogenous cell proliferation after stroke in female rat. J Neurosci Res. 2003; 73(6): 778-786, doi: 10.1002/ jnr.10691, indexed in Pubmed: 12949903.

6. Chopp M, Li Yi. Treatment of neural injury with marrow stromal cells. Lancet Neurol. 2002; 1(2): 92-100, doi: 10.1016/ s1474-4422(02)00040-6, indexed in Pubmed: 12849513.

7. Crigler L, Robey RC, Asawachaicharn A, et al. Human mesenchymal stem cell subpopulations express a variety of neuro-regulatory molecules and promote neuronal cell survival and neuritogenesis. Exp Neurol. 2006; 198(1): 54-64, doi: 10.1016/j.expneurol.2005.10.029, indexed in Pubmed: 16336965.

8. Deng J, Petersen BE, Steindler DA, et al. Mesenchymal stem cells spontaneously express neural proteins in culture and are neurogenic after transplantation. Stem Cells. 2006; 24(4): 1054-1064, doi: 10.1634/stemcells.2005-0370, indexed in Pubmed: 16322639.

9. Emad NG, Safwat WG, Joseph NA, et al. Role of mesenchymal stem cell therapy in cisplatin induced nephrotoxicity in adult albino rats: ultrastructural \& biochemical study. Acta 
Medica International. 2014; 1(2): 57-66, doi: 10.5530/ ami.2014.2.3.

10. Gardoni F, Kamal A, Bellone C, et al. Effects of streptozotocin-diabetes on the hippocampal NMDA receptor complex in rats. J Neurochem. 2002; 80(3): 438-447, doi: 10.1046/j.0022-3042.2001.00713.x, indexed in Pubmed: 11908465.

11. Greco SJ, Zhou C, Ye JH, et al. A method to generate human mesenchymal stem cell-derived neurons which express and are excited by multiple neurotransmitters. Biol Proced Online. 2008; 10: 90-101, doi: 10.1251/bpo147, indexed in Pubmed: 19461957.

12. Jafari Al, Barzegar GH, Pourheidar M. The protective effects of insulin and natural honey against hippocampal cell death in streptozotocin-induced diabetic rats. J Diabetes Res. ; 2014: 491571.

13. Jiang J, Lv Z, Gu Y, et al. Adult rat mesenchymal stem cells differentiate into neuronal-like phenotype and express a variety of neuro-regulatory molecules in vitro. Neurosci Res. 2010; 66(1): 46-52, doi: 10.1016/j.neures.2009.09.1711, indexed in Pubmed: 19808065.

14. Kamal A, Biessels GJ, Urban I, et al. Hippocampal synaptic plasticity in streptozotocin-diabetic rats: impairment of long-term potentiation and facilitation of longterm depression. Neuroscience. 1999; 90(3): 737-745, doi: 10.1016/s0306-4522(98)00485-0.

15. Kim S, Chang KA, Kim Ja, et al. The preventive and therapeutic effects of intravenous human adipose-derived stem cells in Alzheimer's disease mice. PLoS One. 2012; 7(9): e45757, doi: 10.1371/journal.pone.0045757, indexed in Pubmed: 23049854

16. Kumar SK, Perumal S, Rajagopalan V. Therapeutic effect of bone marrow mesenchymal stem cells on cold stress induced changes in the hippocampus of rats. Neural Regen Res. 2014; 9(19): 1740-1744, doi: 10.4103/16735374.143416, indexed in Pubmed: 25422634.

17. Lannert $H$, Hoyer S. Intracerebroventricular administration of streptozotocin causes long-term diminutions in learning and memory abilities and in cerebral energy metabolism in adult rats. Behav Neurosci. 1998; 112(5): 1199-1208, doi: 10.1037//0735-7044.112.5.1199, indexed in Pubmed: 9829797.

18. Lee I, Jerman TS, Kesner RP. Disruption of delayed memory for a sequence of spatial locations following CA1 - or CA3-lesions of the dorsal hippocampus. Neurobiol Learn Mem. 2005; 84(2): 138-147, doi: 10.1016/j. nlm.2005.06.002, indexed in Pubmed: 16054848.

19. Li ZG, Zhang W, Grunberger G, et al. Hippocampal neuronal apoptosis in type 1 diabetes. Brain Res. 2002; 946(2): 221-231, doi: 10.1016/s0006-8993(02)02887-1, indexed in Pubmed: 12137925.

20. Liu L, Xuan C, Shen P, et al. Hippocampal Mechanisms Underlying Impairment in Spatial Learning Long After Establishment of Noise-Induced Hearing Loss in CBA Mice. Front Syst Neurosci. 2018; 12: 35, doi: 10.3389/ fnsys.2018.00035, indexed in Pubmed: 30087600.

21. McEwen BS, Magariños AM, Reagan LP. Studies of hormone action in the hippocampal formation: possible relevance to depression and diabetes. J Psychosom Res. 2002; 53(4): 883-890, doi: 10.1016/s0022-3999(02)00307-0, indexed in Pubmed: 12377298.

22. Magariños AM, McEwen BS. Experimental diabetes in rats causes hippocampal dendritic and synaptic reorganization and increased glucocorticoid reactivity to stress. Proc Natl Acad Sci U S A. 2000; 97(20): 11056-11061, doi: 10.1073/ pnas.97.20.11056, indexed in Pubmed: 11005876.
23. Malone JI, Hanna S, Saporta S, et al. Hyperglycemia not hypoglycemia alters neuronal dendrites and impairs spatial memory. Pediatr Diabetes. 2008; 9(6): 531-539, doi: 10.1111/j.1399-5448.2008.00431.x, indexed in Pubmed: 19067891.

24. Matchynski-Franks JJ, Pappas C, Rossignol J, et al. Mesenchymal Stem Cells as Treatment for Behavioral Deficits and Neuropathology in the 5xFAD Mouse Model of Alzheimer's Disease. Cell Transplant. 2016; 25(4): 687-703, doi: 10.3727/096368916X690818, indexed in Pubmed: 26850119.

25. Mezey E, Key S, Vogelsang G, et al. Transplanted bone marrow generates new neurons in human brains. Proc Natl Acad Sci U S A. 2003; 100(3): 1364-1369, doi: 10.1073/ pnas.0336479100, indexed in Pubmed: 12538864.

26. Munoz JR, Stoutenger BR, Robinson AP, et al. Human stem/ /progenitor cells from bone marrow promote neurogenesis of endogenous neural stem cells in the hippocampus of mice. Proc Natl Acad Sci U S A. 2005; 102(50): 18171-18176, doi: 10.1073/pnas.0508945102, indexed in Pubmed: 16330757.

27. Orlovsky MA, Spiga F, Lebed YV, et al. Early molecular events in the hippocampus of rats with streptozotocin-induced diabetes. Neurophysiology. 2008; 39(6): 435-438, doi: 10.1007/s11062-008-9000-0.

28. Prockop DJ. "Stemness" does not explain the repair of many tissues by mesenchymal stem/multipotent stromal cells (MSCs). Clin Pharmacol Ther. 2007; 82(3): 241-243, doi: 10.1038/sj.clpt.6100313, indexed in Pubmed: 17700588.

29. Revsin Y, Saravia F, Roig P, et al. Neuronal and astroglial alterations in the hippocampus of a mouse model for type 1 diabetes. Brain Res. 2005; 1038(1): 22-31, doi: 10.1016/j. brainres.2004.12.032, indexed in Pubmed: 15748869.

30. Sanchez-Ramos J, Song S, Cardozo-Pelaez F, et al. Adult bone marrow stromal cells differentiate into neural cells in vitro. Exp Neurol. 2000; 164(2): 247-256, doi: 10.1006/ exnr.2000.7389, indexed in Pubmed: 10915564.

31. Schoenle EJ, Schoenle D, Molinari L, et al. Impaired intellectual development in children with type I diabetes: association with $\mathrm{HbA}(1 \mathrm{c})$, age at diagnosis and sex. Diabetologia. 2002; 45(1): 108-114, doi: 10.1007/s125002-8250-6, indexed in Pubmed: 11845229.

32. Vorhees CV, Williams MT. Morris water maze: procedures for assessing spatial and related forms of learning and memory. Nat Protoc. 2006; 1(2): 848-858, doi: 10.1038/ nprot.2006.116, indexed in Pubmed: 17406317.

33. Yang H, Fan S, Song D, et al. Long-term streptozotocin-induced diabetes in rats leads to severe damage of brain blood vessels and neurons via enhanced oxidative stress. Mol Med Rep. 2013; 7(2): 431-440, doi: 10.3892/ mmr.2012.1227, indexed in Pubmed: 23232924.

34. Ye M, Wang XJ, Zhang YH, et al. Therapeutic effects of differentiated bone marrow stromal cell transplantation on rat models of Parkinson's disease. Parkinsonism Relat Disord. 2007; 13(1): 44-49, doi: 10.1016/j.parkreldis.2006.07.013, indexed in Pubmed: 17005432.

35. Zhang $H$, Huang $Z$, Xu Y, et al. Differentiation and neurological benefit of the mesenchymal stem cells transplanted into the rat brain following intracerebral hemorrhage. Neurol Res. 2006; 28(1): 104-112, doi: 10.1179/016164106X91960, indexed in Pubmed: 16464372

36. Zhao F, Li J, Mo L, et al. Changes in neurons and synapses in hippocampus of streptozotocin-induced type 1 diabetes rats: a stereological investigation. Anat Rec (Hoboken). 2016; 299(9): 1174-1183, doi: 10.1002/ar.23344, indexed in Pubmed: 27064698. 